\title{
Top-down and bottom-up approaches to improve educational quality: their intended and unintended consequences
}

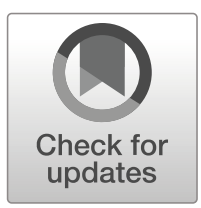

\section{Guri Skedsmo ${ }^{1,2} \cdot$ Stephan Gerhard Huber ${ }^{3}$}

Published online: 9 March 2019

(C) Springer Nature B.V. 2019

One of the main topics covered by this journal during the last three years is teacher assessment and evaluation, which has been put forward as an important strategy for assuring and developing educational quality in many countries, particularly the USA (please see Skedsmo and Huber 2018). This is also an important topic in this issue, which focuses on approaches and models to improve teaching and overall school practice, including in schools in socially disadvantaged areas. Moreover, the importance of involving practitioners' perspectives in such approaches is addressed, as are questions related to the validity of instruments across different educational contexts and possible consequences underperformance may have for the school climate.

\section{Articles in this issue of EAEA, 1/2019}

In the first article, Van den Ouweland, Vanhoof and Van den Bossche report on a qualitative study on the impact underperforming teachers can have on co-workers and how co-workers respond to underperformance. Previous studies conducted in the education and other work sectors have mostly used surveys or experiments, incorporating hypothetical cases. While these studies have focused on co-workers' intentions or attitudes, the authors in this paper aimed to study a real-life incident, in which respondents in the Flemish education context were confronted with an underperforming teacher. Using the critical incident technique, they generated knowledge about how the

Guri Skedsmo

guri.skedsmo@phsz.ch

Stephan Gerhard Huber

stephan.huber@phzg.ch

1 Institute for Research on Professions and Professional Learning, Schwyz University of Teacher Education, Goldau, Switzerland

2 Department of Teacher Education and School Research, University of Oslo, Oslo, Norway

3 Institute for the Management and Economics of Education, University of Teacher Education Zug, Zug, Switzerland 
respondents understood an incident that was important to them, considering cognitive, affective and behavioural elements as well as contextual issues. Generally, underperformance affects co-workers emotionally in the sense that it creates feelings of injustice, particularly in cases where students are affected. Relationships with the underperforming teacher, perceptions of the school's climate, team collaboration and ways in which school leaders are expected to handle these situations are important factors in teachers' considerations of whether to speak up or remain silent about these types of incidents.

In the second article, Paufler and Clark examine the perceptions of school- and district-level administrators regarding a newly established teacher evaluation system in a large, fast-growing suburban school district in the State of Arizona that was awarded 25 million dollars of federal funding from the Race to the Top programme in 2011. The teacher evaluation system comprised two weighted components: a student achievement measure and a professional practice measure (Danielson's Framework for Teaching). The administrators in this study were responsible for evaluating the teachers, and the authors' aim was to gain knowledge about their lived experiences regarding the practices of evaluating teacher quality. Based on the analysis of quantitative and qualitative data, the findings show that even if serious concerns were raised regarding the validity of value-added data and the reliability of classroom observations, most of the administrators did not call for significant changes to the system's design. Overall, they discouraged using the data for high-stakes decisions and, at the same time, strongly supported helping teachers to improve their professional practice, even if this help was based on data produced from imperfect measures. The authors point to several implications and argue that more research is needed on how to develop and implement valid and reliable systems, the results of which can be used by various stakeholders in meaningful ways.

In the third article, Liu, Bell, Jones and McCaffrey analyse the use of Danielson's Framework for Teaching in three different settings and find great variation with respect to several aspects, such as the observation system in which the framework is embedded, what aspects of teaching are rated, the number of raters and lessons rated, the distribution of observation scores and the average teaching quality. As such, they demonstrate that in many educational contexts, the prevailing idea that the validity of an instrument will follow across contexts is misguided, even if the use of an observation system is implemented in similar ways. Not only do their analyses support the claim that different observation systems might produce different basic psychometric validity evidence but they also point to challenges related to interpreting variation across the three cases. Moreover, due to complexity, they suggest that the observation systems and their attendant uses should be the unit of analysis for validation efforts, not the instruments.

In the fourth article, Arnold-Berkovits, Kurz and Reddy investigate the convergent validity of the My Instructional Learning Opportunities Guidance System (MyiLOGS), a teacher self-report log designed to measure students' opportunity to learn an intended curriculum. The MyiLOGS is compared with the Classroom Strategies Assessment System-Observer Form, an online multidimensional assessment of instruction and behaviour management practices. The study included 71 teachers and 22 principals from six high-poverty charter schools in NJ, USA. According to the authors, the findings show initial evidence for the convergent validity of the MyiLOGS with principal ratings through classroom observation, and the combination of the teachers' 
self-report logs and classroom observations could provide useful information to improve teachers' practice.

In the fifth article, Kyriakides, Charalambous, Creemers, Antoniou, Devine, Papastylianou and Fahie present the results of a European study that investigated the extent to which the dynamic approach to school improvement (DASI) can help schools situated in socially disadvantaged areas improve their effectiveness. The study included a sample of 72 primary schools in four European countries (Cyprus, England, Greece and Ireland), selected for their prioritisation of the promotion of equity. The DASI is based on a framework that refers to effectiveness factors that, according to research, need to be considered when initiating change at the school level. It simultaneously gives stakeholders a chance to address their own improvement priorities in ways that can be adjusted over time, according to changing needs and contexts. In addition, the schools are supported systematically by an advisory and research team who collaborates with the school to design, implement and evaluate improvement efforts. By using a quasi-experimental design, the authors show that the DASI influenced students' achievements in mathematics in the experimental schools in all four countries. While the effect of socioeconomic status on student achievement remained the same in the experimental schools, it increased in the control schools.

\section{Reflections on the perspectives of the contributions}

Four of the articles in this issue focus on approaches and tools used to measure and improve the effectiveness of teachers and school practices. Based on the findings of the first article, arguments could be put forward in terms of the need to address the evaluation of teachers' work. In this article, Van den Ouweland et al. show how underperforming teachers can influence co-workers and the learning culture of schools in negative ways. However, the arguments put forward by the authors emphasise school leaders' responsibility to handle such challenges among school staff. Moreover, school leaders need to set an agenda to develop a school culture where learning and the collective responsibility of teachers and school leaders to develop and maintain good learning conditions are at the forefront.

In the fourth and fifth articles, Arnold-Berkovits et al. and Kyriakides et al. report on the approaches of studies that include practitioners' perspectives and focus on developing ownership among school actors. In contrast, the models under scrutiny in the second and third articles, by Paufler and Clark and by Liu et al., are initiated on a state level with less opportunity for school actors to influence and adapt the framework and methodology they are based on. In the latter study, the authors report on problems and challenges related to how the models are used in practice. These point to the underlying logic of models that calls for school governing in terms of controlling and surveilling schools, where feedback in the form of the data produced and incentives and sanctions tied to results are supposed to make teachers and school leaders work harder (see Gunter et al. 2016; Mintrop 2004; Fuller 2008). While bottom-up approaches demonstrate positive results with respect to school development and student learning outcomes, more top-down oriented models seem to have a less positive influence. Rather, they seem to 
produce a range of unintended consequences, and perhaps questions could be raised as to whether they are in danger of contributing to the de-professionalisation of teachers.

Publisher's note Springer Nature remains neutral with regard to jurisdictional claims in published maps and institutional affiliations.

\section{References}

Fuller, B. (2008). Liberal learning in centralising states. In B. Fuller, M. K. Henne, \& E. Hannum (Eds.), Strong States, weak schools: the benefits and dilemmas of centralised accountability (Vol. 16). Bingley: Emerald Group.

Gunter, H. M., Grimaldi, E., Hall, D., \& Serpieri, R. (2016). NPM and educational reform in Europe. In H. M. Gunter, E. Grimaldi, D. Hall, \& R. Serpieri (Eds.), New public management and the reform of education. European lessons for policy and practice. London: Routledge.

Mintrop, H. (2004). Schools on probation: how accountability works (and doesn't work). New York: Teachers College Press.

Skedsmo, G., \& Huber, S. G. (2018). Teacher evaluation: the need for valid measures and increased teacher involvement. Educational Assessment, Evaluation and Accountability, 30(1), 1-5. 